DOI: $10.23857 /$ dc.v5i1.913

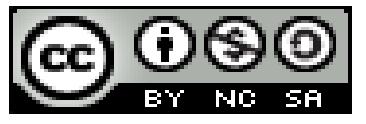

Ciencias de la salud

Artículo de investigación

\title{
Factores de riesgo de la Criptococosis en pacientes con VIH en el hospital de infectología "Dr. José Daniel Rodríguez Maridueña” en el periodo 2015-2016
}

\author{
Risk factors of Cryptococcosis in patients with HIV in the hospital of infectology \\ "Dr. José Daniel Rodríguez Maridueña "in the period 2015-2016
}
Fatores de risco de criptococose em pacientes com HIV no hospital de infectologia "Dr. José Daniel Rodríguez Maridueña "no período 2015-2016

\author{
Glubis W. Gomez-Pelaez ${ }^{\mathrm{I}}$ \\ glubis.gomezp@ug.edu.ec \\ Carlos E. Lara-Zambrano II \\ carlos.laraz@ug.edu.ec \\ Hugo A. Luna-Rodríguez III \\ hugo.lunar@ug.edu.ec \\ Carlos E. Lara-Parra ${ }^{\text {IV }}$ \\ carlos.lara@gamail.com
}

Recibido: 27 de noviembre de 2018 * Corregido: 18 de diciembre de 2018 * Aceptado: 20 de diciembre de 2018

\footnotetext{
I. Magister en Salud Publica; Magister en Nutrición; Especialista en Cirugía General; Doctor en Medicina y Cirugía; Universidad de Guayaquil, Guayaquil, Ecuador.

II. Magister en Salud Publica; Diploma Superior en Enfermedades Inmunodeficientes en VIH-Sida; Especialista en Medicina Interna; Doctor en Medicina y Cirugía; Universidad de Guayaquil, Guayaquil, Ecuador.

III. Especialista en Gerencia y Planificación Estratégica en Salud; Diploma Superior de Cuarto Nivel en Desarrollo Local y Salud; Especialista en Cirugía General; Doctor en Medicina y Cirugía; Universidad de Guayaquil, Guayaquil, Ecuador.

IV. Médico de la Universidad de Guayaquil, Guayaquil, Ecuador.
} 


\section{Resumen}

Antecedentes: La criptococosis es una infección fúngica sistémica que afecta fundamentalmente a pacientes inmunocomprometidos. La incidencia de la infección presentó un aumento aparejado al de la epidemia del síndrome de inmunodeficiencia adquirida (sida) desde su inicio en la década de 1980. La criptococosis se considera una de las micosis oportunistas más importantes en los últimos tiempos; a pesar de esto, en Ecuador son pocos los reportes relacionados con la misma. Métodos: El presente estudio tiene un enfoque cuantitativo de diseño no experimental, de corte transversal y retrospectivo con la utilización del método de observación analítica, cuyo objetivo es proveer información sobre los factores de riesgo de la Criptococosis en personas con VIH. Se revisaron 115 historias clínicas de pacientes con VIH que presentaron Criptococosis y fueron ingresados en el Hospital Dr. José Daniel Rodríguez Maridueña, durante el periodo 2015-2016. Resultados: Se evidenció que la concentración sanguínea menor de $200 \mathrm{CD} 4 \mathrm{cel} / \mathrm{mm} 3$ constituyó el factor más preponderante para la manifestación de la criptococosis, además se pudo constatar que la infección por criptococo se presentó mayormente en la población que habita en áreas Rurales.

Palabras claves: VIH, criptococosis, factores de riesgo.

\section{Abstract}

Background: Cryptococcosis is a systemic fungal infection that primarily affects immunocompromised patients. The incidence of infection increased as a result of the epidemic of acquired immunodeficiency syndrome (AIDS) since its inception in the 1980s. Cryptococcosis is considered one of the most important opportunistic mycoses in recent times; Despite this, in Ecuador there are few reports related to it. Methods: The present study has a quantitative approach of non-experimental cross-sectional and retrospective design with the use of the analytical observation method for a wide information on the subject, whose objective is to provide information on the risk factors of Cryptococcosis in People with HIV. We reviewed 115 medical records of patients with HIV who presented Cryptococcosis and were admitted to the Dr. José Daniel Rodríguez Maridueña Hospital during the period 2015-2016. Results: It was evidenced that the blood concentration of less than $200 \mathrm{CD} 4$ cells / mm3 was the most important factor for the manifestation of cryptococcosis, and it was possible to verify that cryptococcal infection was present mainly in the population living in rural areas. 
Key words: HIV, cryptococcosis, risk factors.

\section{Resumo}

Fundamento: A criptococose é uma infecção fúngica sistêmica que afeta principalmente pacientes imunocomprometidos. A incidência de infecção aumentou como resultado da epidemia da síndrome da imunodeficiência adquirida (AIDS) desde a sua criação na década de 1980. A criptococose é considerada uma das mais importantes micoses oportunistas nos últimos tempos; Apesar disso, no Equador há poucos relatos relacionados a ele. Métodos: O presente estudo tem uma abordagem quantitativa de delineamento transversal não-experimental e retrospectivo com o uso do método de observação analítica para uma ampla informação sobre o assunto, cujo objetivo é fornecer informações sobre os fatores de risco da criptococose em pessoas com HIV. Foram revisados 115 prontuários de pacientes com HIV que apresentaram criptococose e foram internados no Hospital Dr. José Daniel Rodríguez Maridueña durante o período de 2015-2016. Resultados: Evidenciou-se que a concentração sanguínea inferior a 200 células CD4 / mm3 foi o fator mais importante para a manifestação da criptococose, sendo possível constatar que a infecção criptocócica estava presente principalmente na população do meio rural.

Palavras chave: HIV, criptococose, fatores de risco.

\section{Introducción.}

La criptococosis es una infección fúngica sistémica que afecta, fundamentalmente, a pacientes inmunocomprometidos. La misma resulta de la inhalación de esporas del complejo de especies Cryptococcus neoformans para el cual se reconocen, en la actualidad, siete especies de vida libre en la naturaleza. Estas levaduras presentan especial tropismo por el sistema nervioso central. Esto justifica que entre el 70 y $90 \%$ de los pacientes manifiesten cuadros clínicos de meningitis o meningoencefalitis subaguda; no obstante, sus síntomas suelen ser inespecíficos. Según los reportes, ocasiona mortalidad en el $60 \%$ de las personas afectadas a pesar del tratamiento antifúngico

\footnotetext{
738 Vol. 5, núm. 1, enero 2019, pp. 736-750

Glubis W. Gomez-Pelaez; Carlos E. Lara-Zambrano; Hugo A. Luna-Rodríguez; Carlos E. LaraParra
} 
convencional y puede alcanzar el $100 \%$ en los pacientes no tratados o con tratamientos iniciados tardíamente ${ }^{1-6}$.

La incidencia de la infección presentó un aumento aparejado al de la epidemia del síndrome de inmunodeficiencia adquirida (sida) desde su inicio en la década de 1980.5 Adicionalmente, otras condiciones tales como los tratamientos inmunosupresores y los trasplantes, también son consideradas factores predisponentes para el desarrollo de la enfermedad. La criptococosis se considera una de las micosis oportunistas más importantes en los últimos tiempos; a pesar de esto, en Ecuador son pocos los reportes relacionados con la misma ${ }^{6-10}$.

El propósito de este trabajo de investigación es el estudio de los factores de riesgo y características de la Criptococosis en 115 pacientes con VIH atendidos en el Hospital de Infectología Dr. José Daniel Rodríguez Maridueña, durante el periodo 2015-2016, a través de un análisis profundo de las historias clínicas que permitan ver la evolución de los pacientes y el desarrollo de las manifestaciones clínicas mediante un estudio observacional, el cual se evaluara sus complicaciones, sintomatología, cuadro clínico, diagnóstico y tratamiento, este trabajo cumplirá el objetivo de informar acerca de esta enfermedad oportunista común en pacientes con VIH a nivel local $^{11-12}$.

\section{Metodología.}

El presente estudio de investigación tiene un enfoque cuantitativo de diseño no experimental, de corte transversal y retrospectivo con la utilización del método de observación analítica, para una amplia información sobre el tema, cuyo objetivo es proveer información sobre los factores de riesgo de la Criptococosis en personas con VIH.

\footnotetext{
739 Vol. 5, núm. 1, enero 2019, pp. 736-750

Glubis W. Gomez-Pelaez; Carlos E. Lara-Zambrano; Hugo A. Luna-Rodríguez; Carlos E. Lara- 
Se revisaron 115 historias clínicas de pacientes con VIH que presentaron Criptococosis y fueron ingresados en el Hospital Dr. José Daniel Rodríguez Maridueña, durante el periodo 20152016.

\section{Universo y muestra}

Corresponde a los 115 pacientes con VIH que presentaron Criptococosis y fueron ingresados en el Hospital Dr. José Daniel Rodríguez Maridueña, durante el periodo 2015-2016.

\section{Viabilidad}

Este trabajo de investigación es un estudio viable porque tiene la aprobación del Director del Hospital de Infectología Dr. José Daniel Rodríguez Maridueña, de los representantes de la Universidad de Guayaquil para su ejecución, que permitieron el acceso a la evaluación del estado de los pacientes VIH. Es de interés para un grupo de médicos especialistas del área de Infectología por ser casos muy frecuentes en la institución, para que existan datos estadísticos que demuestren la prevalencia de la Criptococosis, se permitirá estudiar los factores de riesgo de la Criptococosis del paciente con VIH, sus complicaciones, su diagnóstico, sintomatología y tratamiento que se les brindo. Este trabajo es autofinanciado por el autor.

\section{Resultados.}

Casos reportados con VIH+ criptococosis en años 2015-2016.

\footnotetext{
740 Vol. 5, núm. 1, enero 2019, pp. 736-750
Glubis W. Gomez-Pelaez; Carlos E. Lara-Zambrano; Hugo A. Luna-Rodríguez; Carlos E. LaraParra
} 


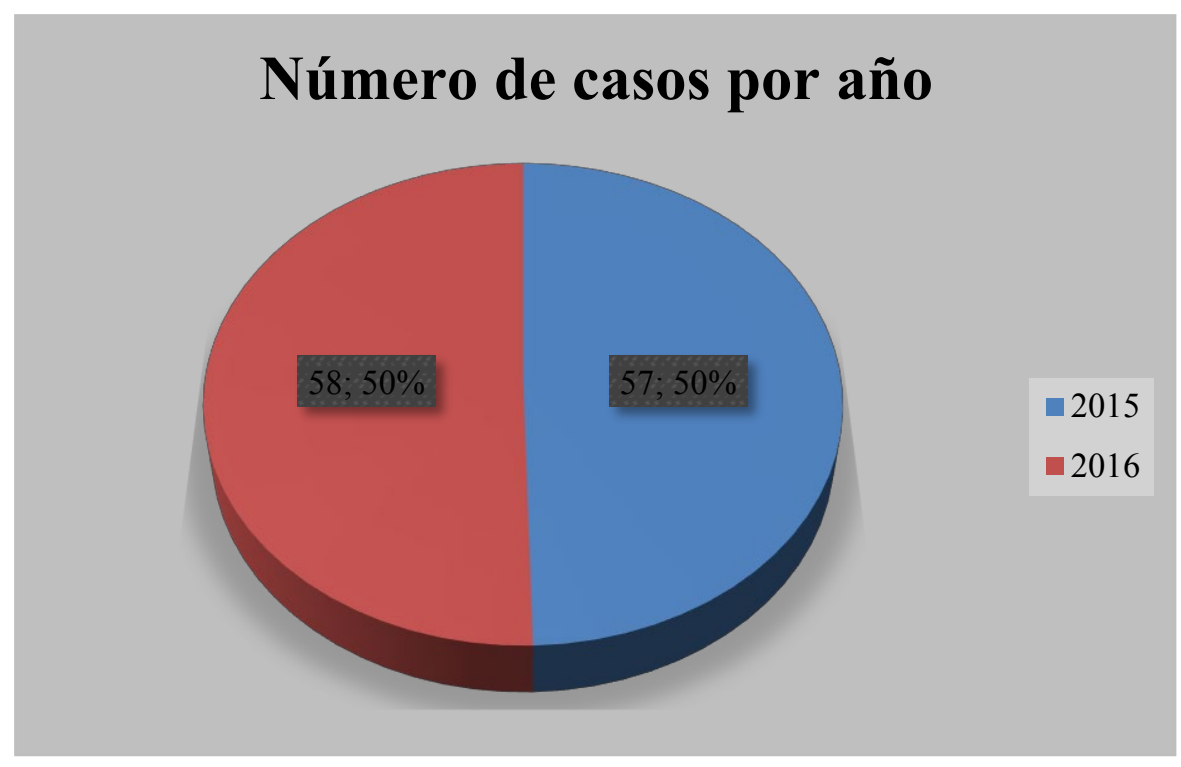

Figura $N^{\circ}$ 1.- Casos por año de criptococosis en el periodo 2015-2016

Interpretación: Número de casos confirmados de criptococosis en el periodo 2015-2016, la incidencia es similar en ambos años. 
Factores de riesgo de la Criptococosis en pacientes con VIH en el hospital de infectología "Dr. José Daniel

Formas de presentaciones clínicas de la Criptococosis

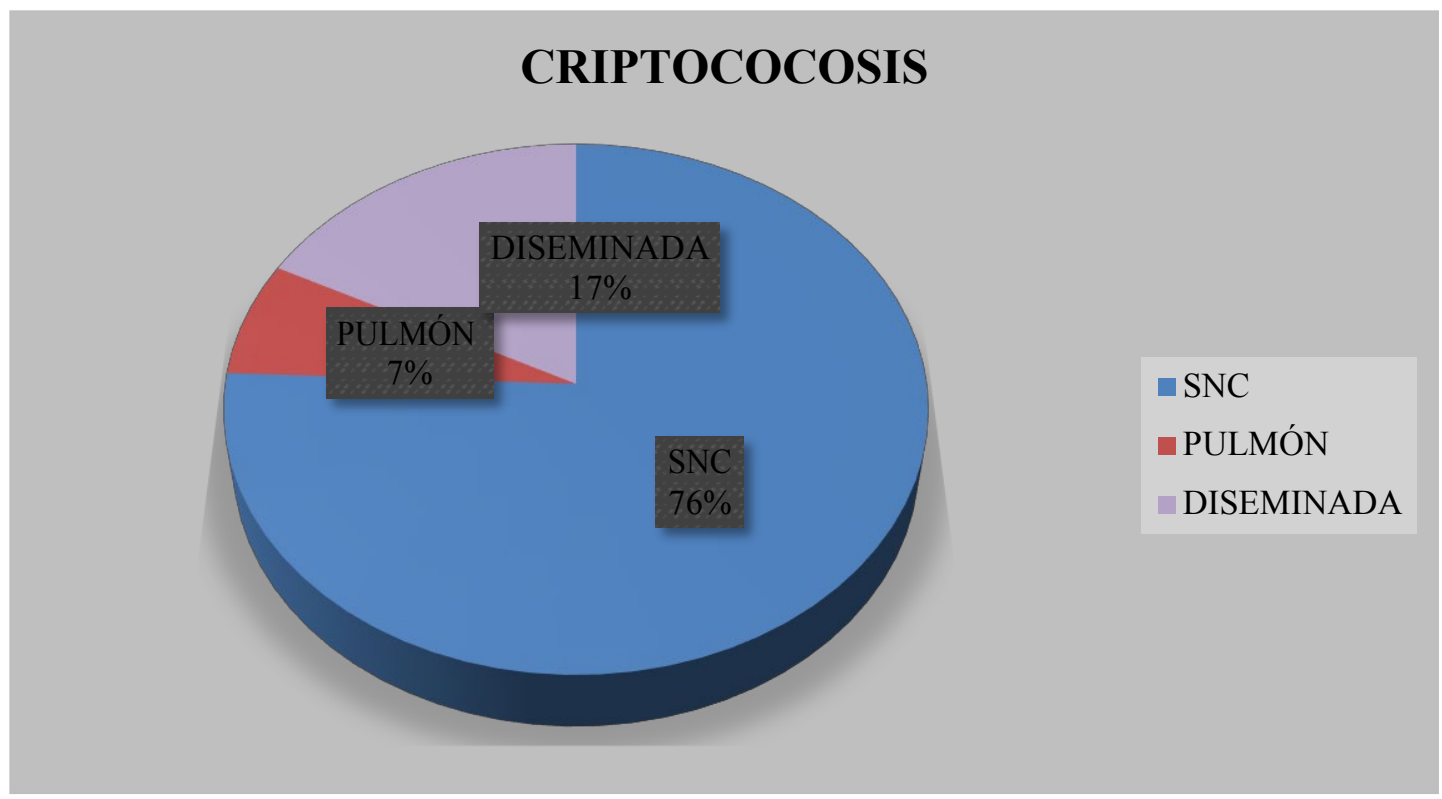

Figura $N^{\circ}$ 2.- Formas de presentación clínica de la criptococosis en el hospital de infectología

Interpretación: En el 76\% de casos, la criptococosis se manifestó en el sistema nervioso central demostrando el tropismo del hongo hacia esa zona del organismo, seguido de la presentación diseminada y pulmonar. 
Factores de riesgo de la Criptococosis en pacientes con VIH en el hospital de infectología "Dr. José Daniel

Casos reportados con VIH-criptococosis de acuerdo al sexo

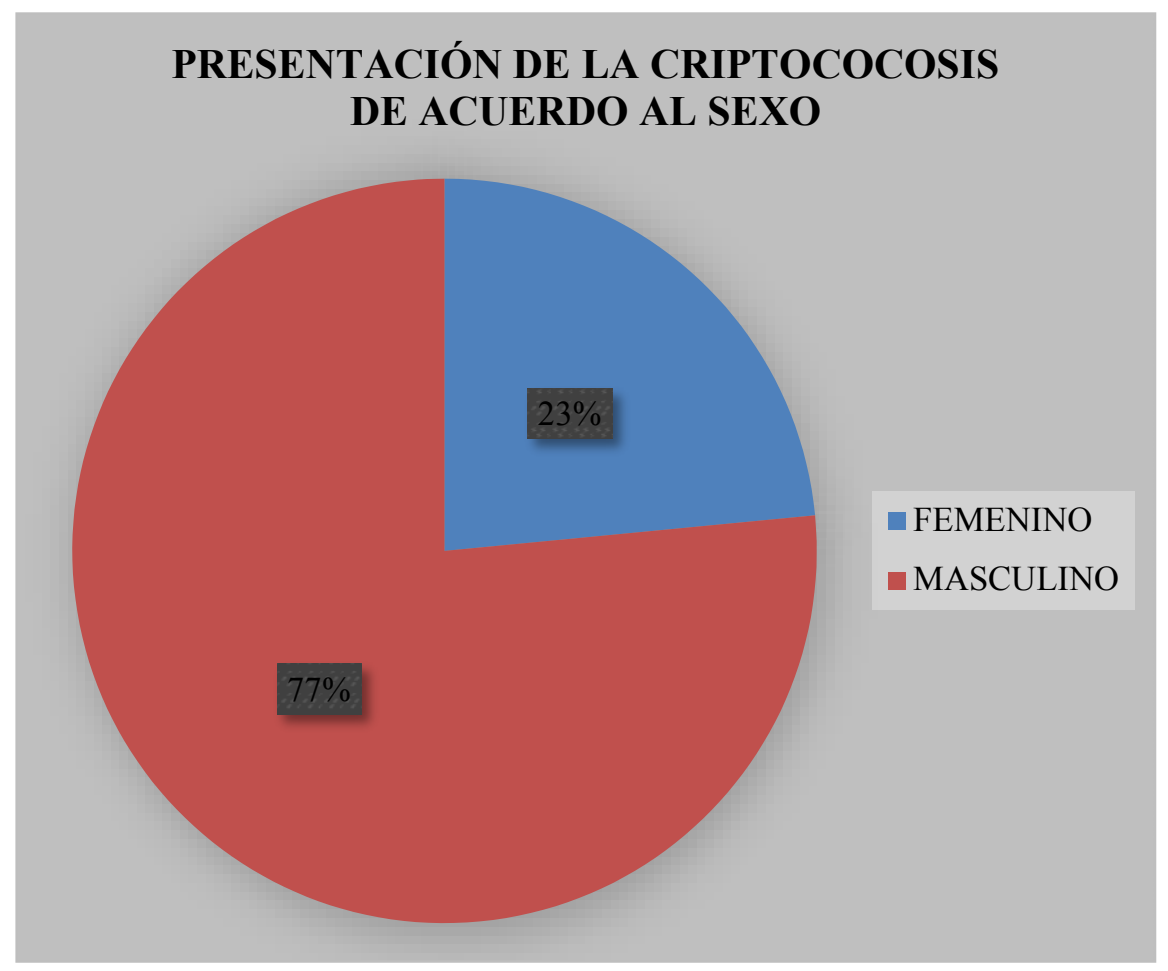

Figura $N^{\circ}$ 3.- Casos reportados con criptococosis clasificados de acuerdo al sexo

Interpretación: La criptococosis se presentó mayormente en el sexo masculino. 
Factores de riesgo de la Criptococosis en pacientes con VIH en el hospital de infectología "Dr. José Daniel

Casos reportados con VIH-criptococosis de acuerdo a edad

\section{PRESENTACIÓN DE CRIPTOCOCOSIS POR EDAD}

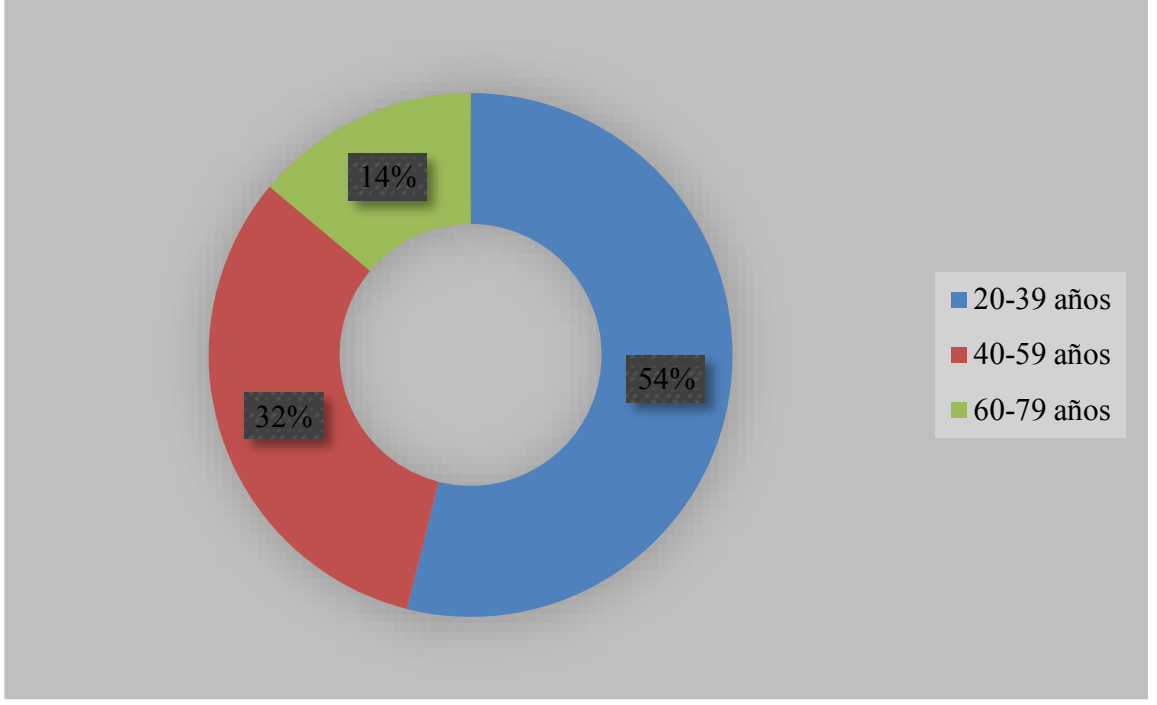

Figura $N^{\circ}$ 4.- Casos reportados con criptococosis clasificados de acuerdo a la edad

Interpretación: El grupo etario en el que se presentó mayormente la criptococosis fue entre los 2039 años, con un $54 \%$ de los casos. 
Factores de riesgo de la Criptococosis en pacientes con VIH en el hospital de infectología "Dr. José Daniel

Zona de procedencia de los pacientes diagnosticados con $\mathrm{VIH}+$ criptococosis

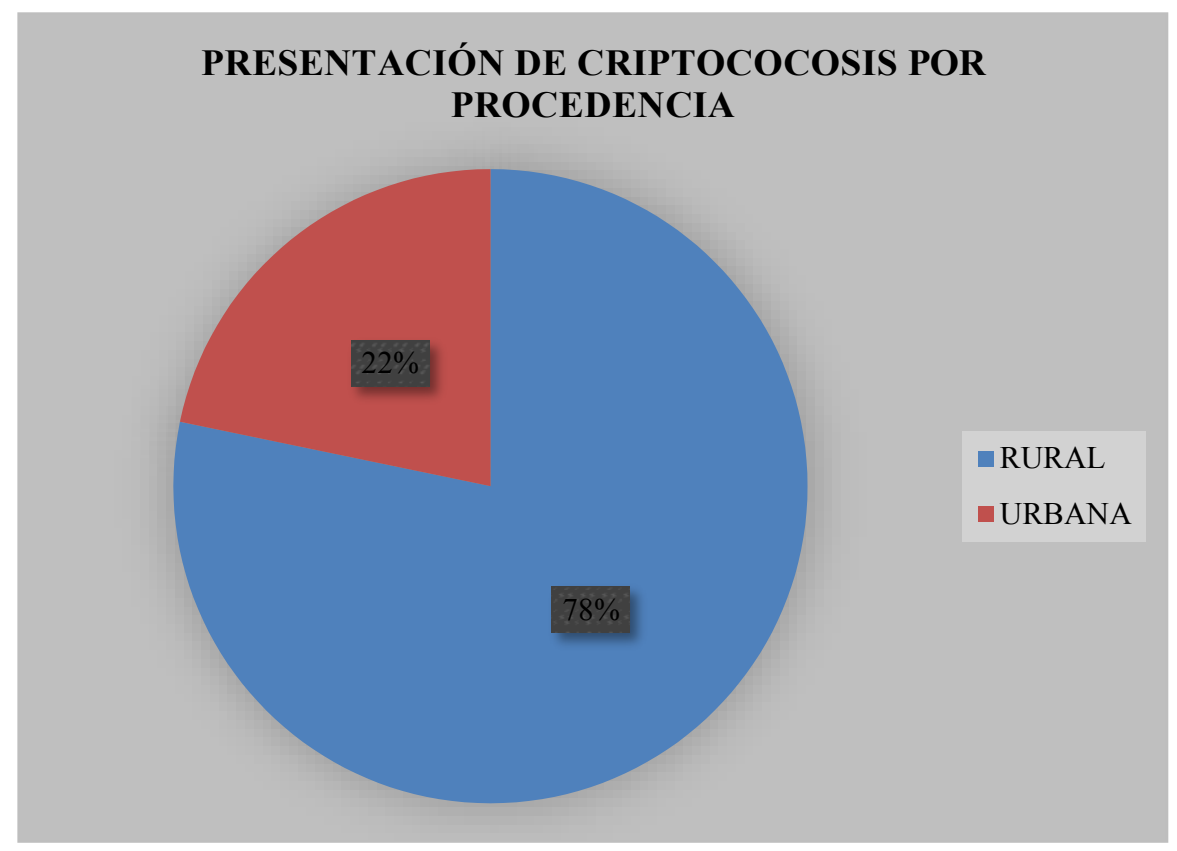

Figura $N^{\circ}$ 5.- Zona de procedencia de los pacientes diagnosticados con VIH+CRIPTOCOCOSIS

Interpretación: El porcentaje mayoritario de la criptococosis se presentó en la población rural con un $78 \%$, lo que indica el mayor contacto que tienen los habitantes de esta zona con las esporas del hongo. 
Factores de riesgo de la Criptococosis en pacientes con VIH en el hospital de infectología "Dr. José Daniel

Concentración de células cd4 en sangre al momento del diagnóstico de VIH+criptococosis

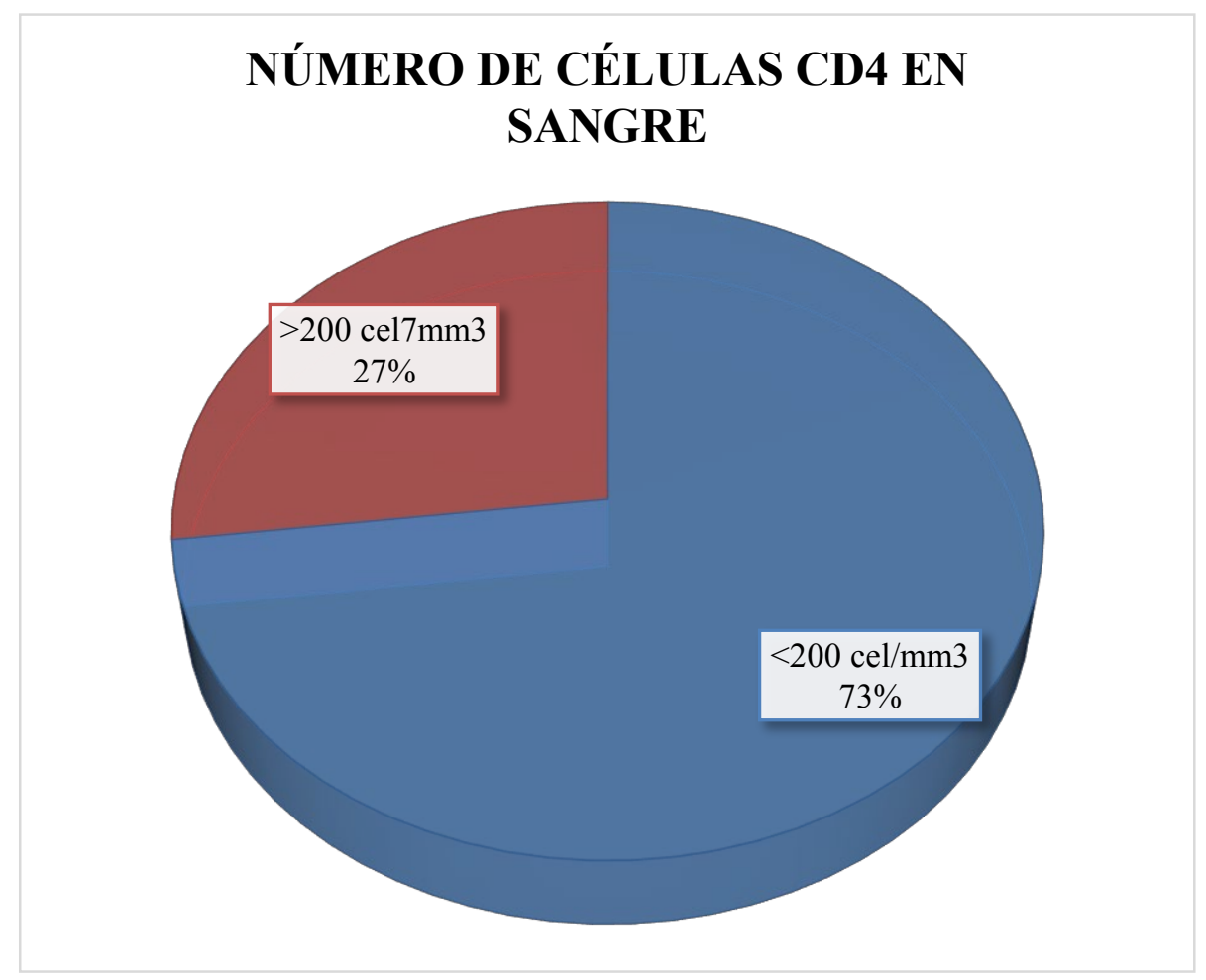

Figura $\mathrm{N}^{\circ}$ 6.- Concentración sanguínea de células al momento del diagnóstico de criptococosis

Interpretación: Siendo la criptococosis una enfermedad oportunista el número de células CD4 en sangre constituye un factor preponderante para la presentación de la misma, lo que se demuestra en este estudio, en el cual el $73 \%$ de pacientes tenía menos de $200 \mathrm{cel} / \mathrm{mm} 3$. 
Factores de riesgo de la Criptococosis en pacientes con VIH en el hospital de infectología "Dr. José Daniel Rodríguez Maridueña” en el periodo 2015-2016

Reporte de casos de VIH+ criptococosis de acuerdo a egreso hospitalario en el año 2015

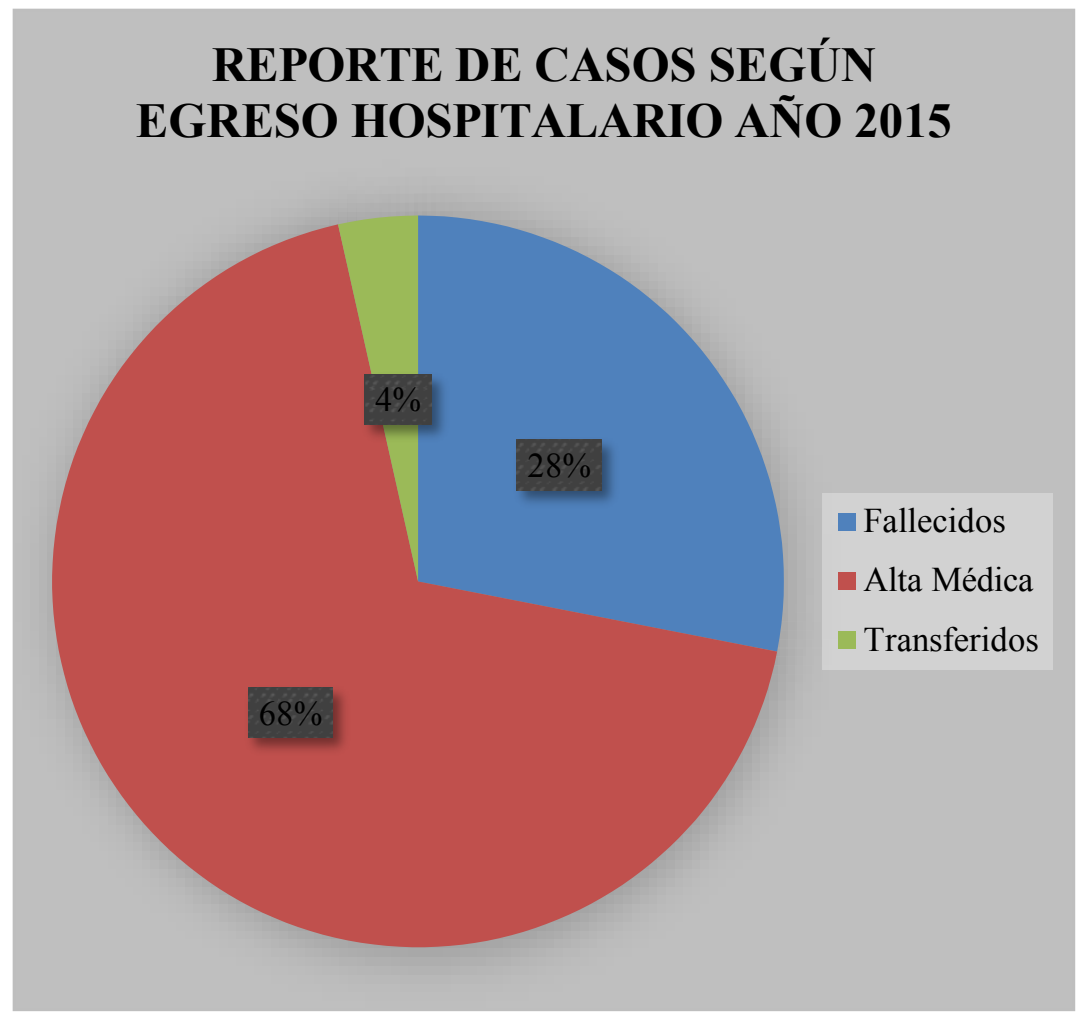

Figura $N^{\circ}$ 7.- Casos de VIH+ criptococosis de acuerdo a egreso hospitalario en el año 2015

Interpretación: En el año 2015 el 28\% de pacientes con Vih+criptococosis que fue atendido en el hospital de infectología falleció debido a sus complicaciones. 
Factores de riesgo de la Criptococosis en pacientes con VIH en el hospital de infectología "Dr. José Daniel Rodríguez Maridueña” en el periodo 2015-2016

Reporte de casos de VIH+ criptococosis de acuerdo a egreso hospitalario en el año 2016

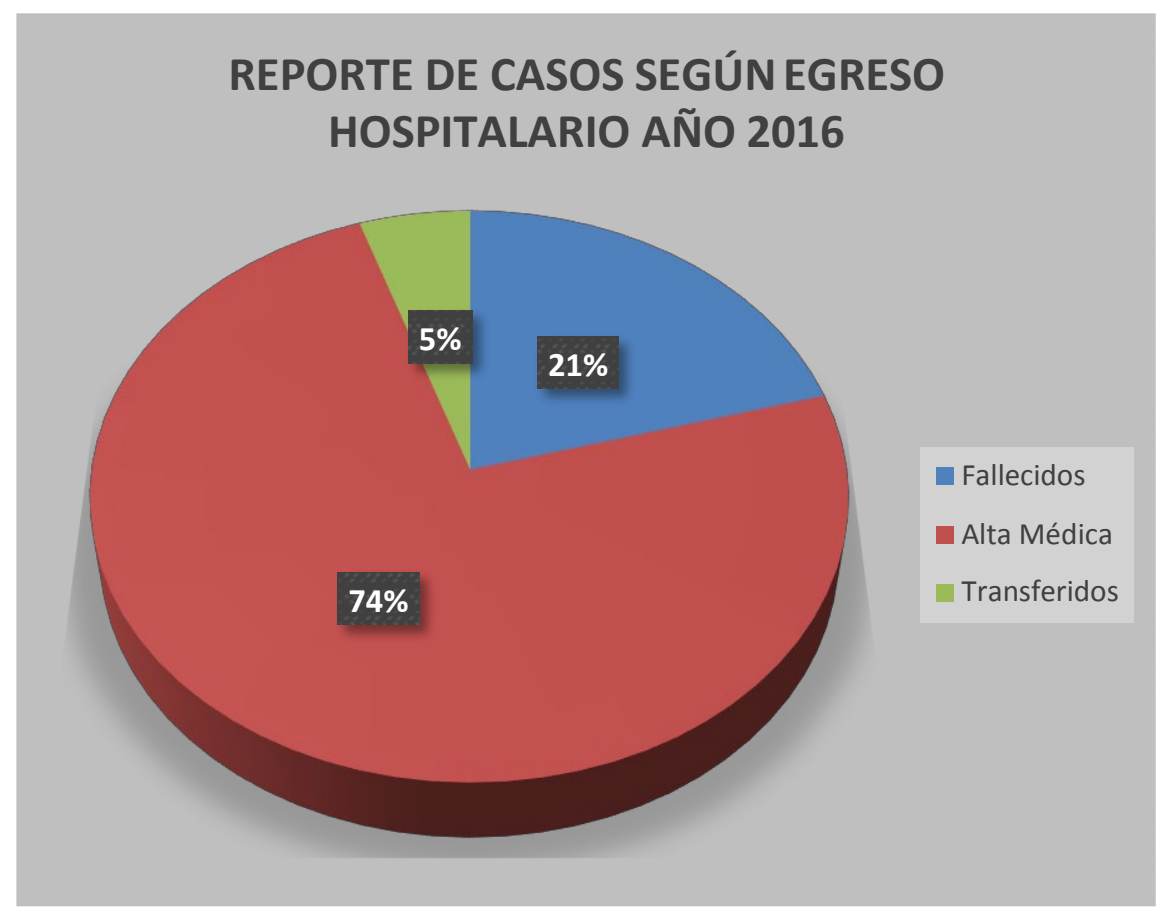

Figura $\mathrm{N}^{\circ}$ 8.- Casos de $\mathrm{VIH}+$ criptococosis de acuerdo a egreso hospitalario en el 2016

Interpretación: En el año 2016 el porcentaje de fallecidos fue de $21 \%$ un poco menor al del año anterior.

\section{Conclusiones.}

El presente estudio ha tenido como objetivo determinar qué factores de riesgo predisponen al desarrollo de la infección por criptococo en pacientes VIH que ingresaron al Hospital de infectologia Dr. Daniel Rodríguez Maridueña, se encontró que la aparición de la enfermedad tiene asociación con el deficiente estado inmunológico, reflejado en el número de células CD4, evidenciándose en la mayoría de los pacientes una concentración en sangre $<200$ cel/mm3 CD4. 
Se demostró además que la población rural es donde se presenta más comúnmente la criptococosis,

lo que presupone el mayor contacto que tienen los habitantes de dicha zona con los nichos en que se desarrolla el criptococo.

La edad y el sexo no demostraron tener relación con la presentación de la micosis.

\section{Bibliografía.}

1. Padilla Desgarennes MdC, Navarrete Franco G, Pérez Cortés S. Criptococosis diseminada asociada con VIH. Dermatologia Revista Médica Mexicana. 2012 Oct; 53(2): p. 126-131.

2. Nucci M QTFCAyc. www.bago.com. [Online].; 2010. Available from: http://www.bago.com/BagoArg/Biblio/infectoweb585.htm

3. Lorena JM. Manifestaciones neurológicas del virus VIH. Rev. "Medicina" Vol. 15 N 2. 2010;: p. 139.

4. Tomás Baró MT. Epidemiologia de la Criptococosis en España. [Online].; 2012. Available from: http://www.tdx.cat/bitstream/handle/10803/3864/mtbt1de2.pdf?sequence=1.

5. Tircio Esparza SS. Determinación de criptococosis en pacientes con vih factores de riesgo y complicaciones en el hospital de infectologia "Dr Jose Daniel Rodriguez Maridueña" en el periodo 2013-2014. Trabajo de titulación presentado como requisito para optar por el grado de médico. Guayaquil: Universidad de Guayaquil, Facultad de Ciencias Médicas; 2015.

6. Torres M. Cuidado de la Salud. [Online].; 2014. Available from: http://www.cuidadodelasalud.com/salud/que-es-la-enfermedad-de-criptococosis/.

7. Tangarife C. Aprende en línea. [Online].; 2011. Available from: http://aprendeenlinea.udea.edu.co/lms/moodle/mod/page/view.php?id=100866.

8. Tello M, Gutiérrez E, Béjar V, Galarza C, Ramos W. Criptococosis. Rev. Méd. Risaralda. 2013 Sep; 19(2): p. 147-153.

9. Alan M, Sugar M. www.merckmanuals.com. [Online].; 2016. Available from: http://www.merckmanuals.com/es-pr/professional/enfermedadesinfecciosas/hongos/criptococosis.

10. Paúl SCD. Criptococosis en pacientes con SIDA: manifestaciones cutáneas y sistémicas, diagnóstico y terapéutica. Revista CES Medicina. 2010; 10(2): p. 79-89. 
Factores de riesgo de la Criptococosis en pacientes con VIH en el hospital de infectología “Dr. José Daniel Rodríguez Maridueña” en el periodo 2015-2016

11. Trombetta L. Manifestaciones clínicas de la criptococosis asociada al Sida. Revista Panamericana de Infectologia. 2010 Mar; 43(1): p. 13-16.

12. ADAM. CLINICA DAM. [Online].; 2016. Available from: https://webcache.googleusercontent.com/search?q=cache:Cgkw4UiG3ikJ:https://www.clinicada $\mathrm{m} . \mathrm{com} / \mathrm{salud} / 5 / 001328 . \mathrm{html}+\& \mathrm{~cd}=8 \& \mathrm{hl}=\mathrm{es}-419 \& \mathrm{ct}=\mathrm{clnk} \& \mathrm{gl}=\mathrm{ec}$. 